\title{
Preliminary Investigation on Coupling MCDA with GLUE to Perform Uncertainty Analysis of a Hydrological Model
}

\author{
P. Vallam, X. S. Qin, and J. J. Yu
}

\begin{abstract}
Hydrological models are being used for different applications. Quantifying the uncertainty of popular Hydrological models has been well documented, especially with Bayesian methods such as the Generalized Likelihood Uncertainty Estimation (GLUE). However, research studies have often either neglected the lesser known hydrological models or have performed a typical Bayesian analysis of uncertainty. In this paper, the SLURP model's uncertainty is examined using a novel approach of the GLUE method. Instead of considering the overall Nash Sutcliffe Efficiency (NSE), the NSE values of different magnitudes of flows are considered simultaneously to capture the predictive uncertainties of the SLURP model. By using a Multi-Criterion Decision Analysis (MCDA) method, the NSE values of different flow periods are simultaneously considered when computing the predictive intervals of the SLURP model. Also, the potential issues of using a MCDA based GLUE approach in lieu of the traditional GLUE approach are discussed.
\end{abstract}

Index Terms-TOPSIS, MCDA, bayesian, GLUE.

\section{INTRODUCTION}

Modeling in Hydrology has expanded beyond traditional uses and is being utilized over a wide spectrum of applications. Traditionally, to ensure that the model is physically representative of the catchment, it is usually calibrated over a period of time. Following the calibration, verification of the model is carried out which enables future use of the model for that catchment. This process was apt when the basis of application was limited to a stand-alone usage. Now, hydrological models are employed in conjunction with other models including atmospheric models, groundwater models, environmental models, etc. Therefore it can be concluded that there is a critical need to understand the nature of the model predictions. This usually is characterized by performing uncertainty analysis of the model output.

There are many different methods employed to perform uncertainty analysis of the model. They are broadly classified into Bayesian and non-Bayesian methods. One popular Bayesian method is the Generalized Likelihood Uncertainty Estimation (GLUE) method. Many researchers have applied it with valid results, which underscore the versatility and robustness of the GLUE method [1]-[3]. The GLUE method is a likelihood estimator based method developed by Beven

Manuscript received March 20, 2014; revised May 21, 2014. This research was supported by Singapore's Ministry of Education (MOE) AcRE Tier 1 Project (M4010973.030) and Tier 2 Project (M4020182.030).

The authors are with the Civil and Environmental Department, Nanyang Technological University, Singapore 637598 (e-mail: pramodh1@e.ntu.edu.sg,xsqin@ntu.edu.sg, yuji0011@e.ntu.edu.sg). and Binley [4] which used Monte Carlo simulation to quantify uncertainty in a model.

The model used in this study is the SLURP model developed by Kite [5]. The model has been used by different studies to model diverse catchments, including large mountainous catchment [6], wetland [7], etc. Hence, SLURP is considered as a robust hydrological model. Another important factor contributing to the decision for choosing SLURP as the model for this study, is the makeup of the model parameters. After carrying out analysis, Kite [5] provides the ten most sensitive parameters that affect the model accuracy. Hence, this makes it a very useful case to determine changes in the quantification of parametric uncertainty when the MCDA method is applied with GLUE.

\section{Study CASE AND Methodology}

\section{A. Case Study}

The Kootenay Catchmentis chosen as the case study to demonstrate the applicability of MCDA in the GLUE framework. The mountainous basin which is located in remote British Columbia, Canada was divided into three sub-catchments or Aggregated Simulated Areas (ASAs). The ASAs are Crossing, Canal, and Skookum (outlet of the waterhsed). The primary land covers of this catchment are Crop/Grass, Forest and Impervious and the characteristics are given in Table I.

\begin{tabular}{|c|c|c|c|c|}
\hline \multicolumn{2}{|l|}{ Variable } & Crossing & Canal & Skookum \\
\hline \multicolumn{2}{|c|}{ Area of ASA $\left(\mathrm{km}^{2}\right)$} & 411 & 5040 & 1790 \\
\hline \multicolumn{2}{|c|}{ Mean Elevation (m) } & 1762 & 1892 & 1769 \\
\hline \multirow{3}{*}{$\begin{array}{l}\text { Land Covers } \\
(\%)\end{array}$} & Impervious & 10 & 9 & 9 \\
\hline & Forest & 74 & 70 & 62 \\
\hline & Crop/Grass & 16 & 21 & 29 \\
\hline
\end{tabular}

Source: Kite [5]

The SLURP model balances the water budget by dividing it into four different reservoirs, the canopy storage, the snow storage, the fast, and slow storage. Further technical details of the case study are provided in Kite [5]. The ten most sensitive parameters and their upper and lower bounds are given in Table II. The calibration process of the model involves 10 years data from 1979 to 1988 (3653 continuous data in a daily time step). This is followed by a two-year verification period from 1989 to 1990. The meteorological data for the corresponding period was obtained from the Canadian Daily Climate Data [8] and from the CFSR database [9]. The hydrometric data at the outfalls of the three ASAs were obtained from Environment Canada's Hydat database [10]. The topographic information was obtained from Kite [5]. 


\section{B. GLUE-MCDA Method}

The GLUE method was developed by Beven and Binley [4]. The underlying principle of this method is that each parameter ensemble is accorded equal probability of being representative of the physical processes subsumed in the SLURP model. Initially, multiple parameter ensembles are created within their known upper and lower bounds. In this study, 100,000 such samples were created using the Latin Hypercube Sampling Scheme to create representative samples. The set of parameters given in Table II are replicated for each land-cover and uniform distribution is used to generate the ensembles. As there are three land-covers in the Catchment, 300,000 samples were created with every 30 samples forming a single parameter ensemble.

TABLE II: SENSITIVE PARAMETERS OF SLURP MODEL

\begin{tabular}{|l|l|l|l|}
\hline Channels & $\begin{array}{l}\text { Call } \\
\text { num. }\end{array}$ & $\begin{array}{l}\text { Lower } \\
\text { Bound }\end{array}$ & $\begin{array}{l}\text { Upper } \\
\text { Bound }\end{array}$ \\
\hline $\begin{array}{l}\text { Initial contents of snow storage } \\
\text { (mm) }\end{array}$ & $\mathrm{P} 1$ & 1 & 1000 \\
\hline Initial contents of slow storage (\%) & $\mathrm{P} 2$ & 0 & 100 \\
\hline $\begin{array}{l}\text { Maximum infiltration rate } \\
\text { (mm/day) }\end{array}$ & $\mathrm{P} 3$ & 10 & 100 \\
\hline Manning's Roughness, n & $\mathrm{P} 4$ & 0.0004 & 0.1 \\
\hline $\begin{array}{l}\text { Retention capacity for fast storage } \\
\text { (day) }\end{array}$ & $\mathrm{P} 5$ & 1 & 50 \\
\hline $\begin{array}{l}\text { Maximum capacity for fast storage } \\
\text { (mm) }\end{array}$ & $\mathrm{P} 6$ & 10 & 500 \\
\hline $\begin{array}{l}\text { Retention capacity for slow } \\
\text { storage (day) }\end{array}$ & $\mathrm{P} 7$ & 10 & 300 \\
\hline $\begin{array}{l}\text { Maximum capacity for slow } \\
\text { storage (mm) }\end{array}$ & $\mathrm{P} 8$ & 100 & 1000 \\
\hline Precipitation factor & $\mathrm{P} 9$ & 0.8 & 1.5 \\
\hline $\begin{array}{l}\text { Rain/snow division temperature } \\
\text { (ㄷ) }\end{array}$ & $\mathrm{P} 10$ & -2 & 2 \\
\hline Source: Kite [5] & & \\
\hline
\end{tabular}

Source: Kite [5]

After identification of the most sensitive parameters, a suitable likelihood function is chosen. The likelihood function indicates the performance of the parameter ensemble during the verification period. In this study, the commonly used Nash Sutcliffe Efficiency (NSE) is used. NSE which calculates the accuracy of the model predictions is commonly used in the applications of the GLUE method [3].

The threshold level is set at 0.75 , which entails that all parameter ensembles which generate a NSE value greater than 0.75 is deemed to be physically representative of the hydrological processes in the Catchment. The successful values of the parameters maybe further analyzed to determine their attributes. The model outputs of all the successful parameters are analyzed by matching the confidence intervals with the observed flow.

The MCDA method used in this study is the Technique for Order Preference by Similarity to Ideal Solution (TOPSIS). This MCDA method was first developed by Hwang and Yoon [11]. The methodology of TOPSIS entails the calculation of indices to determine distance from the ideal solution and the negative ideal solution. Therefore, the best performing solution would possess the farthest distance to the negative ideal solution and the shortest distance to the positive ideal solution. The TOPSIS methodology as detailed by Triantaphyllou and Lin [12] is employed. In this study, the flows of the catchment are classified into different categories and the NSE value for each of the category is calculated for each parameter ensemble. Then the indices are cutoff at a point to analyze and compare the flows with the results from GLUE and with other variations of GLUE-MCDA methodologies.

\section{RESULTS AND DISCUSSIONS}

In the GLUE methodology, the 100,000 parameter ensembles were run within the SLURP framework using the Matlab platform. Of the 100,000 parameter ensembles, 4238 different parameter ensembles were considered to have crossed the threshold. The results from applying the GLUE methodology are depicted in Fig. 1. The upper bounds of the confidence interval (CI) are accurate when compared with the observed flow. The lower bounds of the CI however are less accurate when compared with the observed flow in the Kootenay Catchment. Different methodologies instrumented were chosen to overcome these shortcomings and study the shifting of the CIs.

\section{A. Methodology I}

When applying the GLUE-MCDA method, a critical issue in the methodology pertains to the classification of flows. The process to divide the flows into different categories is arbitrary and requires thorough investigation. The first option considered entailed carrying out the B17 flood frequency analysis for the catchment. The HEC-SSP 2.0 software from the USACE was utilized for this task.

The median plotting positions and the computed probability curve for the Kootenay Catchment are depicted in Fig. 2. By using the Return periods of 1, 2 and 10 years, the flows were classified into different categories. The different categories were flows $<400 \mathrm{~m}^{3} / \mathrm{sec}, 400-640 \mathrm{~m}^{3} / \mathrm{sec}, 640-867$ $\mathrm{m}^{3} / \mathrm{sec}$ and $>867 \mathrm{~m}^{3} / \mathrm{sec}$. The NSE value for each category was calculated and finally, the TOPSIS procedure was performed to develop the indices. Equal weight age was given for all the flow categories. The ideal solution was considered to be the maximum NSE value for each category and the non-ideal solution was the minimum NSE value for the same category. To ensure parity when comparing the outcomes of the GLUE-MCDA and just the GLUE methodology, equal width in the TOPSIS index and the likelihood function was considered. The maximum and minimum overall NSE values were -0.2985 and 0.8291 respectively. With these values, the distance to the threshold value of 0.75 was calculated to be 0.0702 . Similarly, the minimum and the maximum value of the calculated TOPSIS index were examined and the same procedure performed to arrive at the new cutoff point. With a minimum and maximum TOPSIS index for the segmented NSE values being 0.3969 and 0.9048 , the cutoff point was computed to be 0.8692 . This yielded the top 6 values for further analysis. The confidence intervals of these predictions were calculated and subsequently plotted in Fig. 3. The results presented a significant savings in the time required for computation when compared to the GLUE methodology (Fig. 1). However, the TOPSIS method performed in this manner compromised on the accuracy of the Confidence Intervals of the flow, especially for the first year. The difference in the nature of the hydrographs for the two years played an important role in the output of the methodology. The hydrograph for the second 
year possessed multiple peaks and this methodology is definitely applicable to perform a cursory analysis on the uncertainty in flows. Another important point of similarity in the results is the comparable magnitudes of the lower bounds for both the results and the observed flow during the low flow periods.

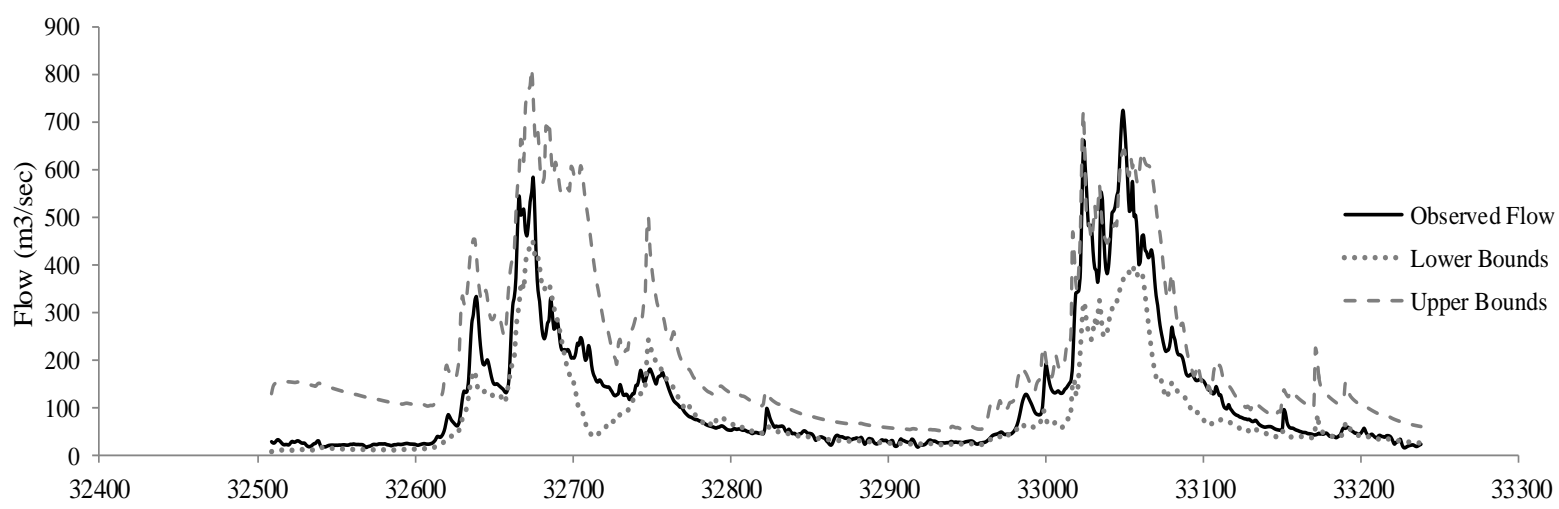

Fig. 1. CI of the flows during 1989-1990 by applying the GLUE methodology.

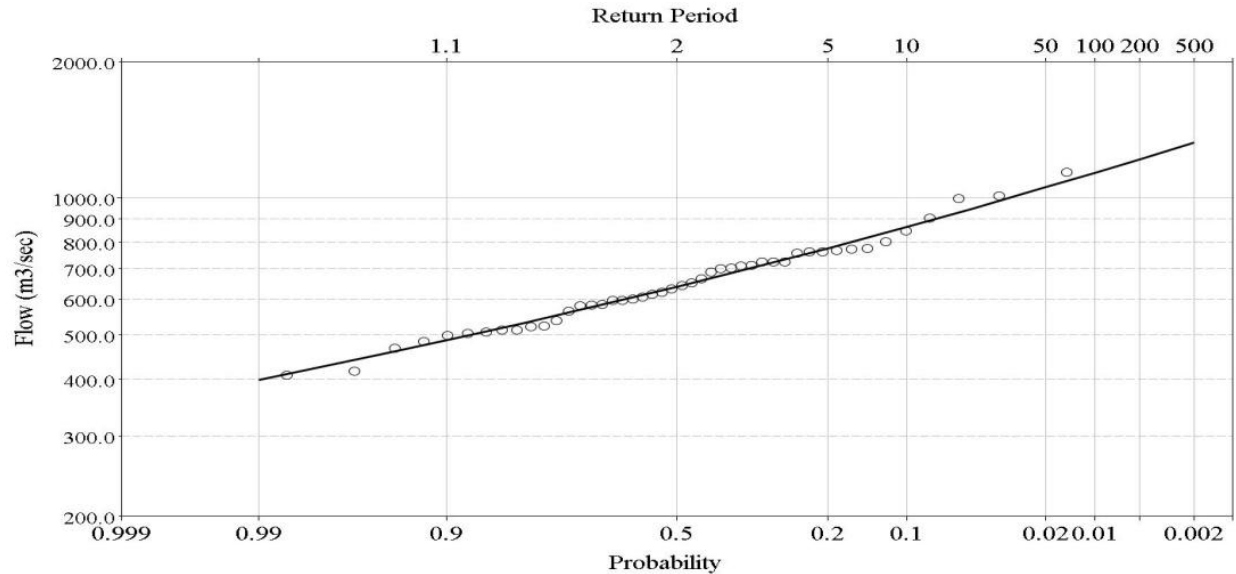

Fig. 2. B17 Plot for the Kootenay basin.

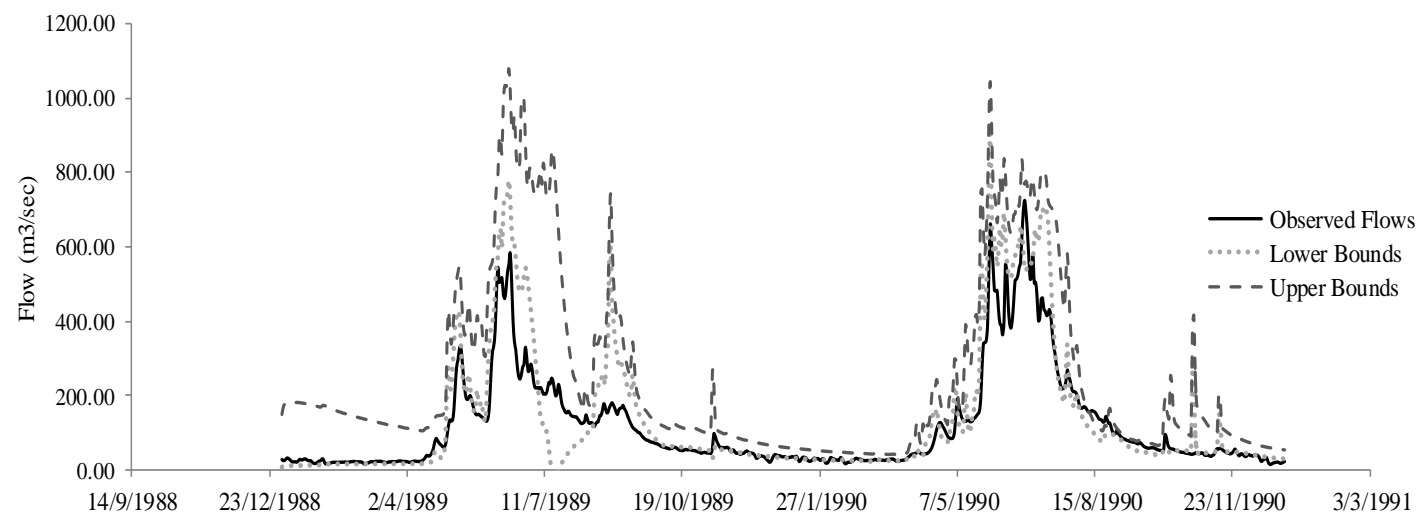

Fig. 3. CI of the flows during 1989-1990 by applying the GLUE-MCDA Methodology I.

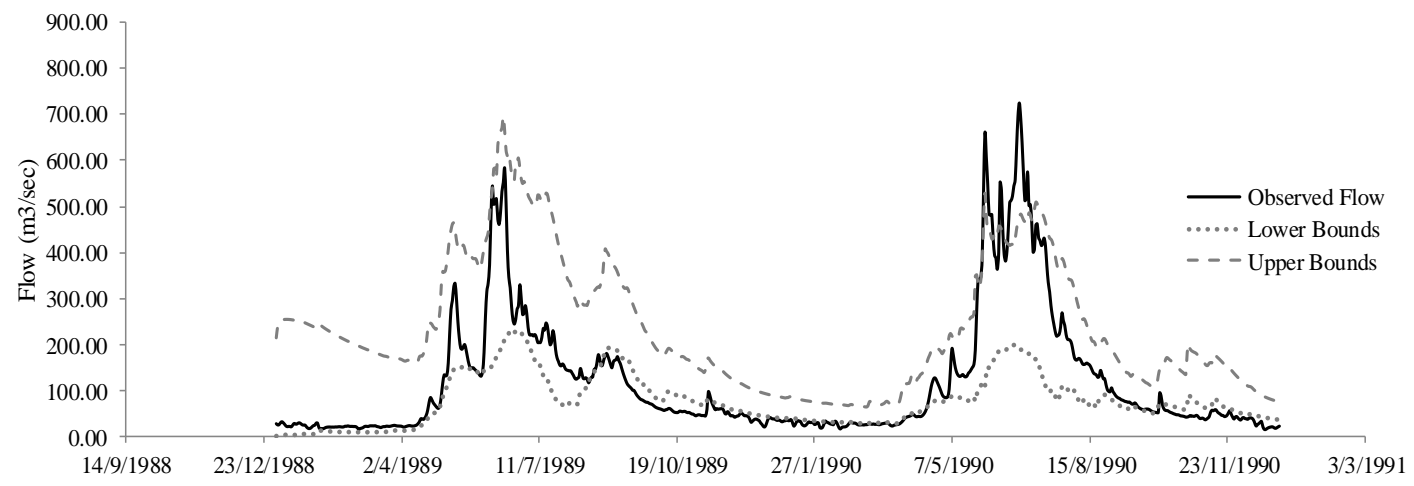

Fig. 4. CI of the flows during 1989-1990 by applying the GLUE-MCDA Methodology II. 


\section{B. Methodology II}

Following this, the flow categories were rationalized to ensure improvements over the previous methodology. The categories were adjusted to equally represent the different magnitudes in the flows observed in Kootenay Catchment. The categories then adopted were $<100 \mathrm{~m}^{3} / \mathrm{sec}, 100-250$ $\mathrm{m}^{3} / \mathrm{sec}, 250-450 \mathrm{~m}^{3} / \mathrm{sec}, 450-600 \mathrm{~m}^{3} / \mathrm{sec}$ and $>600 \mathrm{~m}^{3} / \mathrm{sec}$.

The NSE values were given equal weights of 0.2 when TOPSIS methodology was performed. As with the earlier case, the indices were computed and the equal width was used as the cutoff point. The highest and the lowest TOPSIS indices were 0.9419 and 0.4225 . The output from the top 7130 parameter ensembles were then selected to generate the Confidence Intervals. Fig. 4 depicts the CI for the flows generated in this manner.
The CI from this methodology presented an improvement over the results from the previous methodology. The CI of the peak flows during the first year is more balanced when compared to the GLUE methodology (Fig. 1) and previous methodology (Fig. 3). This is of critical importance when the magnitude of the upper bound is closer to the actual flow. This would make this methodology more suitable for future modifications of the river flow based on the peak availability. However, the drawback of this method manifests when the peak flows of the second year are examined. The SLURP model predicts hydrographs of a single major peak more accurately than hydrographs with multiple peaks for this watershed. This is most evident in the current methodology where the categorization of flows pre-disposes the accuracy of the flows towards flows of lower magnitudes.

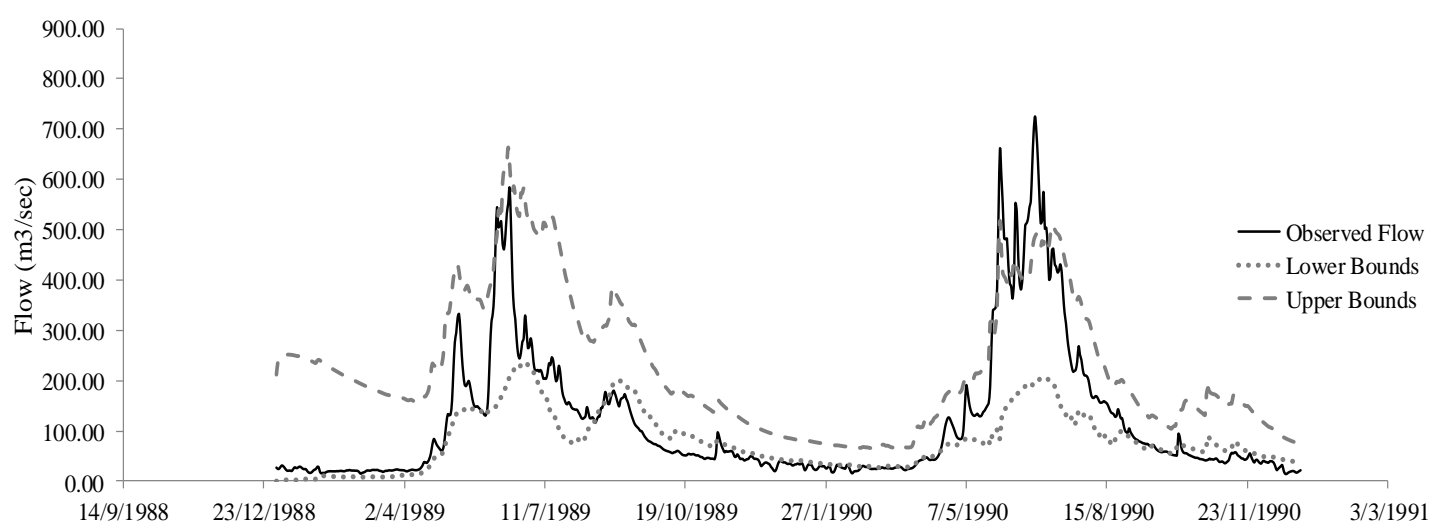

Fig. 5. CI of the flows during 1989-1990 by applying the GLUE-MCDA Methodology III.

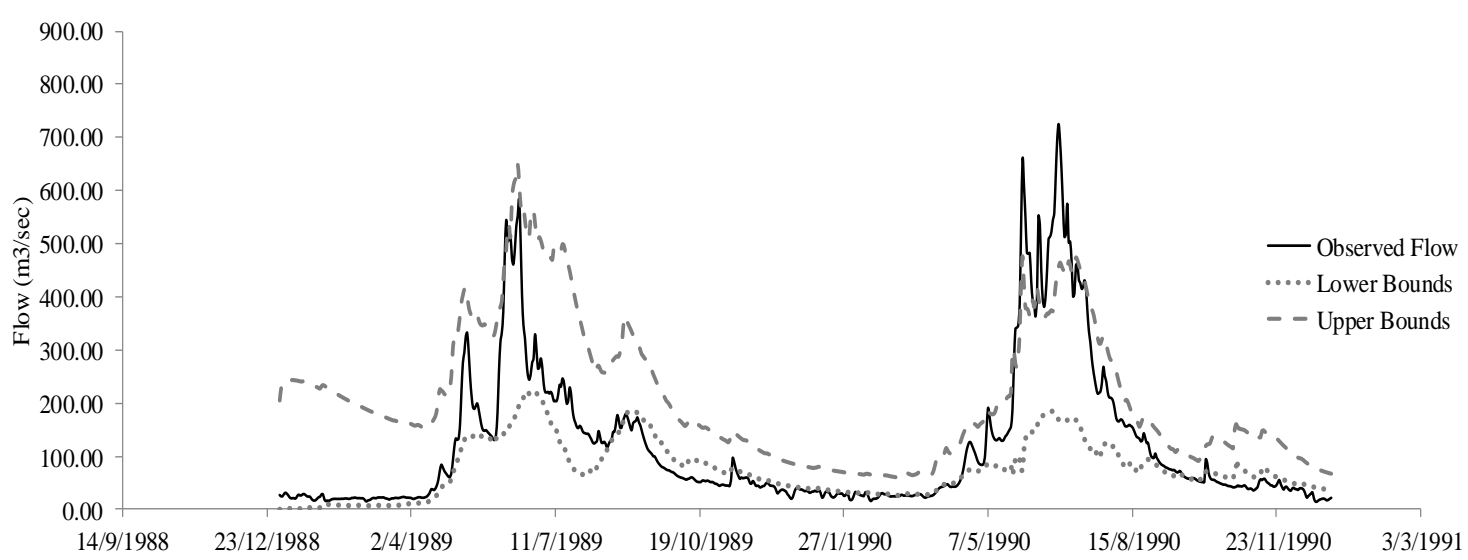

Fig. 6. CI of the flows during 1989-1990 by applying the GLUE-MCDA Methodology IV with weights $0.33,0.33$, and 0.34.

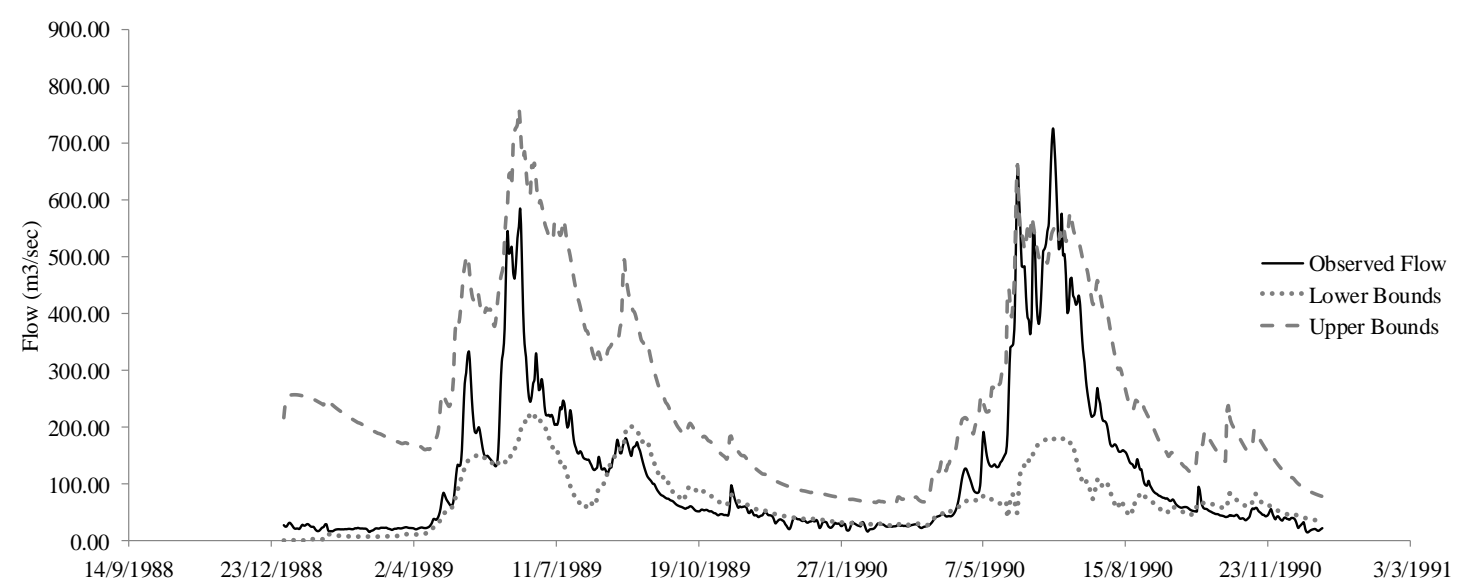

Fig. 7. CI of the flows during 1989-1990 by applying the GLUE-MCDA Methodology IV with weights $0.1,0.1$, and 0.8 . 


\section{Methodology III}

Another aspect of the methodology to understand the variations of the CI of the flows is to quantify the influence of the overall NSE within the TOPSIS framework. Uncertainty quantification by computing and further analyzing the CI of the flows can be modified to suit the research purposes. In this part of the GLUE-MCDA methodology, the overall NSE is accorded a weighing factor of 0.3 and the different categories are accorded a weighing factor of 0.15 . The exception was the $>600 \mathrm{~m}^{3} / \mathrm{sec}$ category which was accorded a weighing factor of 0.1 on the account that only a few periods of flows were modeled in this category. Based on maximum and minimum TOPSIS index values of 0.9385 and 0.4173 , the cutoff point was 0.8837 which resulted in the top 36171 parameter ensembles performing. The CI of the flows (Fig. 5) depicts the reduction of magnitudes of the upper limit of the CI during the peak of the first year. During the low flow periods, the model generally tended to predict flows greater than the observed flow. This property of the SLURP model was observed when performing analyses with all the different versions of the methodologies. This may be attributed to the model structure that predisposes predictions towards lagging in output.

\section{Methodology IV}

After examining the results from the previous two methodologies, it can be concluded that the GLUE-MCDA methodology is quite sensitive to the categorization of the flows. Another aspect of the methodology that affects the accuracy is the weights accorded to the NSE of each category. To further rationalize the flow categories, the magnitudes were divided into low flow, medium flow and peak flow. The categories were $<100 \mathrm{~m}^{3} / \mathrm{sec}, 100-350 \mathrm{~m}^{3} / \mathrm{sec}$ and $>350 \mathrm{~m}^{3} / \mathrm{sec}$. To study the influence of the weights accorded to the NSE of each category, weights of $0.33,0.33$ and 0.34 were initially accorded to each category. Following this, a weight of 0.8 was accorded to one category and 0.1 to the other categories. Altering of weights for each category can typify the shifting of CIs of flows predicted in a typical uncertainty analyses.

The 100,000 parameter ensembles were run with the weights $0.33,0.33$ and 0.34 and the TOPSIS indices calculated. Based on the principle of equal width, the cutoff point for the TOPSIS indices was computed as 0.9136. This yielded 25221 best performing samples. The CIs of these flows were computed and plotted in Fig. 6. The magnitudes of the upper bounds in the results depicted by Fig. 6 demonstrate significantly better performance for the first year when compared to the GLUE methodology. The two most significant improvements is the reduction in magnitudes of the upper CI limits and the non-exceedence of the lower CI limits during the peak flow periods. However, as the CI width of this period is narrower when the GLUE methodology is applied, the application of the GLUE methodology is considered to be more appropriate. During the second year, the CIs of the flows were shown to under-predict for the catchment. This can be attributed to the majority of the flows in the 730 time steps ( 2 years) belonging to the low flow period.

The weights of 0.8 for low flow period and weights of 0.1 and 0.1 were assigned to the medium and high flow periods.
Based on the principle of equal width, the cutoff point for the TOPSIS indices was computed as 0.8962 (which yielded the top 33197 ensembles). Similarly the cutoff points were 0.8982 when medium flow was accorded 0.8 (yielding 67056 ensembles) and 0.9010 when high flow was accorded 0.8 (yielding 65452 ensembles). The results of these three different variations of the methodology yielded expected results. There was an increase in the magnitudes of the upper bounds of the $\mathrm{CI}$ of the peak flows when the high flow was accorded 0.8 (Fig. 7). However significant change in the lower bounds of the CI was noticed during the peak flow period. The upper bounds of the CI for the second year were observed to be closer to the observed flow when high flow was accorded a weight of 0.8 , making this methodology more appropriate for studies focusing on the peak flow events in the catchment. For studies focusing on the low flow periods, such as irrigation or drought management studies, assigning low flow category with a weight higher than 0.8 may be more suitable to obtain a weighed and balanced view of the catchment.

\section{CONCLUSIONS}

The different methodologies discussed in the previous section illustrate the various directions in which the GLUE-MCDA methodology may be applied. The final results present potential improvements in certain aspects of the flows over the different variations of the methodology. However, the results from the GLUE methodology are considered to be more accurate, especially when the second year is considered. An attempt to investigate the salient factors that affect the accuracy of the overall framework has been documented. By suitably modifying this methodology, uncertainty quantification focusing on the different aspects of hydrologic modeling can be conducted, enhancing the role of MCDA methods in traditional uncertainty analyses. The most suitable and comparable result of the methodology would usually require the inclusion of the overall NSE. Moreover, from the results obtained, it can be judged that the results based solely on the different categories may still yield less accurate results when compared to the GLUE methodology. Furthermore, including the GLUE technique within the MCDA framework demonstrates potential to enhance the validity of the uncertainty analyses, modifications to the MCDA framework beyond the flow categories maybe essential.

\section{REFERENCES}

[1] Z. Y. Shen, L. Chen, and T. Chen, "Analysis of parameter uncertainty in hydrological and sediment modeling using GLUE method: A case study of SWAT model applied to Three Gorges Reservoir Region, China," Hydrol. and Earth Sys. Sci., vol. 16, no. 1, pp. 121-132, January 2012

[2] A. Montanari, "Large sample behaviors of the generalized likelihood uncertainty estimation (GLUE) in assessing the uncertainty in rainfall-runoff simulations," Water Resour. Research, vol. 41, no. 8, pp. 1-13, August 2005.

[3] J. R. Stedinger, R. M. Vogel, S. U. Lee, and R. Batchelder, "Appraisal of the generalized likelihood uncertainty estimation," in Proc. World Environment and Water Resources Congress, Honolulu, May 2008, pp. W00B06-1-W00B06-17.

[4] K. Beven and A. Binley, "The future of distributed models: Model calibration and uncertainty prediction," Hydrol. Processes, vol. 6, no. 3, pp. 279-298, July/September 1992. 
[5] G. W. Kite, Manual for the SLURP Hydrological Model V. 11, Saskatoon, Canada: NHRC, 1997.

[6] R. Thorne and M. K. Woo, "Efficacy of a hydrologic model in simulating discharge from a large mountainous catchment," Journal of Hydrol., vol. 330, no. 1-2, pp. 301-312, October 2006.

[7] K. D. Voogt, G. W. Kite, P. Droogers, and H. Murray-Rust, "Modeling water allocation between a wetland and irrigated agriculture in the Gediz Basin, Turkey," Intl. Journal of Water Resour. Develpmt., vol. 16 , no. 4, pp. 639-650, 2000.

[8] National Climate Data and Information Archive. (2014). Historical climate data-environment Canada, Gatineau, QC. [Online]. Available: http://weather.gc.ca/

[9] National Centers for Environmental Prediction (NCEP) Climate Forecast System Reanalysis (CFSR). (2013). Global Weather for SWAT. [Online]. Available: http://globalweather.tamu.edu/

[10] National Water Data Archive. (2012). HYDAT archived hydrometric data, environment Canada, Gatineau, QC. [Online]. Available: http://www.wsc.ec.gc.ca/applications/H2O/index-eng.cfm

[11] C. L. Hwang and K. Yoon, Multiple Attribute Decision Making: Methods and Applications, New York: Springer-Verlag, 1981.

[12] E. Triantaphyllou and C. T. Lin, "Development and evaluation of five fuzzy Multiattribute decision-making methods," Intl. Journal of Approx. Reasoning, vol. 14, no. 4, pp. 281-310, May 1996.

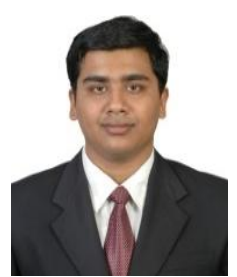

Pramodh Vallam received M.S. degree in environmental engineering from Stanford University and Nanyang Technological University, Singapore in 2010.

$\mathrm{He}$ is currently a Ph.D. candidate in environment and water resources engineering in Nanyang Technological University. He is working on the application of hydrological models to model large-scale watersheds. He was a project officer with the DHI-NTU Water \& Environment Research Center and Education Hub, DHI Water \& Environment (S) Pte. Ltd., working on a sustainability project for the Nanyang Technological University. His research focuses on hydrology, water resources planning, uncertainty quantification and impact of climate change on large-scale watersheds.

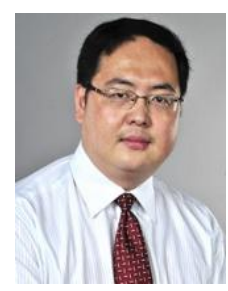

Xiaosheng Qin received Ph.D. degree in environment system engineering from University of Regina, Canada in 2008.

$\mathrm{He}$ is an assistant professor at the Nanyang Technological University, Singapore. His research focuses on water resources planning, hydrologic modeling, climate change impact assessment groundwater modeling, urban air quality and solid waste management.

Dr. Qin was awarded the Young Scientist Paper Award (Third Place) at the 6th International Conference on Environmental Science and Technology 2012, Houston, USA, and also awarded the Best Practice-Oriented Paper Award (American Society of Civil Engineers) from Environmental Multi-Media Council, World Water \& Environmental Resources Congress, Salt Lake City, Utah, USA in June 2004. He is an associate member of ASCE, member of ISEIS, IWA and IAHR

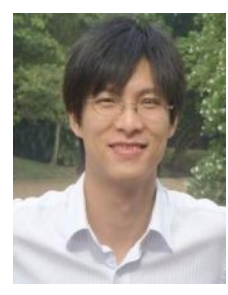

Jianjun Yu received M.Sc. degree in geographic information system in Zhejiang University, China in 2005.

He is currently a researcher in DHI-NTU Water \& Environment Research Center and Education Hub, DHI Water \& Environment (S) Pte. Ltd. His research focuses on hydrology, hydraulics, water resources planning and watershed management, uncertainty analysis, flood and environmental risk management, application of geographic information system and remote sensing techniques in environment and water resources fields. 